\title{
Etude électrophysiologique préliminaire sur la régénération du nerf mentonnier après écrasement chez le rat
}

\section{Electrophysiological preliminary study of mental nerve regeneration after a crush lesion in rat}

\author{
MATHILDE SAVIGNAT *, PHILIPPE LAVALLE **, PHILIPPE LIBERSA *
}

\section{RÉSUMÉ}

La régénération du nerf mentonnier après écrasement et pose d'une membrane, constituée de polyélectrolytes et fonctionnalisée par le Nerve Growth Factor (NGF), est évaluée chez le rat à l'aide d'enregistrements électrophysiologiques. Le NGF semble faciliter la régénération. Med Buccale Chir Buccale 2007; 13: 103106.

mots clés: régénération, nerf mentonnier, facteur de croissance nerveux

\section{SUMMARY}

This preliminary study aims to test if a multilayered polyelectrolyte membrane functionalized with Nerve Growth Factor (NGF) improves the regeneration of the mental nerve after a crush lesion in rat. Neurograms were recorded before lesion, immediately after and one month after the lesion in two groups of injuried rats: one group without membrane $(n=10)$ and one group with the membrane functionalized with NGF wrapped around the wounded nerve $(n=10)$. This novel support seems to facilitate mental nerve regeneration one month after the crush. Med Buccale Chir Buccale 2007; 13: 103-106.

key words: regeneration, mental nerve, nerve growth factor

** Inserm Unité 595, Faculté de Chirurgie dentaire, Université Louis Pasteur, 11 rue Humann, 67085 Strasbourg Cedex 


\section{PROBLEMATIQUE}

Les lésions du nerf alvéolaire inférieur, même minimes, peuvent engendrer une perte de sensibilité dans le territoire d'innervation mais aussi des paresthésies, des dysesthésies et des douleurs chroniques [1,2] mal tolérées par le patient. L'intervention sur le nerf lésé n'est pas toujours indiquée car la majorité des patients retrouve progressivement une sensibilité normale. Cependant, dans certains cas, pour faciliter la régénération, il est indispensable d'intervenir très précocement. Ceci suggère l'utilisation de moyens thérapeutiques peu invasifs, qui seraient adaptés à tous les types de lésions et qui ne risqueraient pas d'endommager davantage le nerf.

Dans ce sens, l'impact d'une membrane, constituée de polyélectrolytes ${ }^{[3]}$ et fonctionnalisée par le Nerve Growth Factor (NGF), sur la régénération médecine buccale chirurgie buccale

VOL. $13, \mathrm{~N}^{\circ} 2$ 2007

page 104 du nerf mentonnier après écrasement, a été testé chez le rat. Le nerf mentonnier a été choisi car il est d'un abord plus aisé et que les proportions des différentes fibres sont similaires à celles du nerf alvéolaire inférieur ${ }^{[4]}$.

\section{MATERIEL ET METHODES}

Deux groupes de rats lésés sont utilisés : le groupe contrôle ou CONT $(n=10)$ où il n'y a pas de pose de membrane et le groupe NGF $(n=10)$ avec pose d'une membrane fonctionnalisée par le NGF.

Les rats sont anesthésiés et le nerf mentonnier mis en évidence. Un neurogramme afférent est enregistré avant la lésion, à l'aide d'une électrode unipolaire ultrafine empalée dans le nerf, de façon à ce que chaque rat soit son propre témoin. Tous les neurogrammes sont enregistrés avec le même protocole de stimulation mécanique $\left(54 \mathrm{mN} / \mathrm{mm}^{2}\right)$ de la commissure labiale. La lésion est créée par un écrasement du nerf juste en avant du foramen mentonnier, elle est objectivée par un enregistrement électrophysiologique. Puis dans le groupe NGF, la membrane $(6 \mathrm{~mm} \times 3 \mathrm{~mm}, 254 \mathrm{ng}$ de NGF) est enroulée autour du nerf à l'endroit de la lésion et fixée par une goutte de colle chirurgicale (Fig. 1). Dans les deux groupes, les tissus sont
Figure 1 : Pose de la membrane autour du nerf mentonnier lésé

Positioning of the membrane around the isolated mental nerve

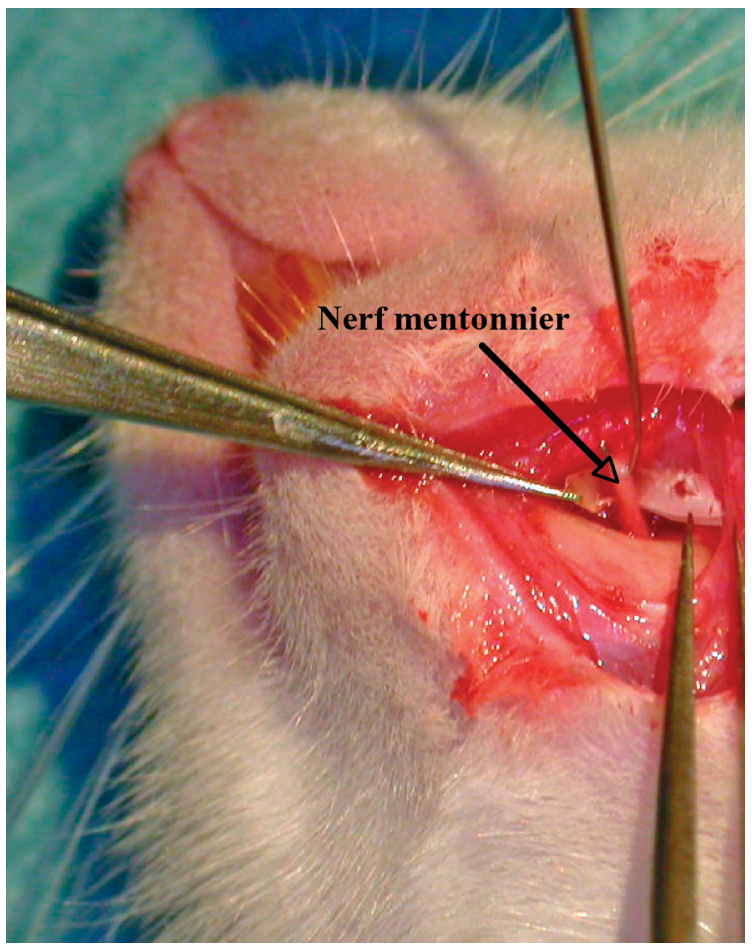

suturés et les rats sont placés 3 jours sous analgésiques et 7 jours sous antibiotiques. Un mois après la lésion, les rats sont opérés selon le même protocole et un neurogramme est enregistré.

\section{RESULTATS PRELIMINAIRES}

Avant la lésion, des bouffées d'activité afférente sont enregistrées après la stimulation. Elles ne se maintiennent pas durant toute la durée de la stimulation. Après la lésion, seul le bruit de fond est enregistré. A un mois, une décharge de base (en l'absence de stimulation) est présente chez certains rats dans les deux groupes, mais plus fréquemment dans le groupe NGF. Dans le groupe CONT, des bouffées d'activité moins amples qu'avant lésion réapparaissent. Dans le groupe NGF, ces bouffées sont plus amples qu'avant lésion et se maintiennent fréquemment pendant toute la durée de la stimulation (Fig. 2). 
Figure 2 : Neurogrammes pour les groupes CONT et NGF : les neurogrammes (amplification 20000) témoignent de l'activité afférente avant lésion $(A)$, immédiatement après $(B)$ et un mois après lésion $(C)$ dans les groupes CONT et NGF

Neurograms in groups CONT and NGF : afferent neurogram activity, amplified 20000, before lesion (A), immediately after (B), and one month after crush lesion (C) in CONT and NGF groups

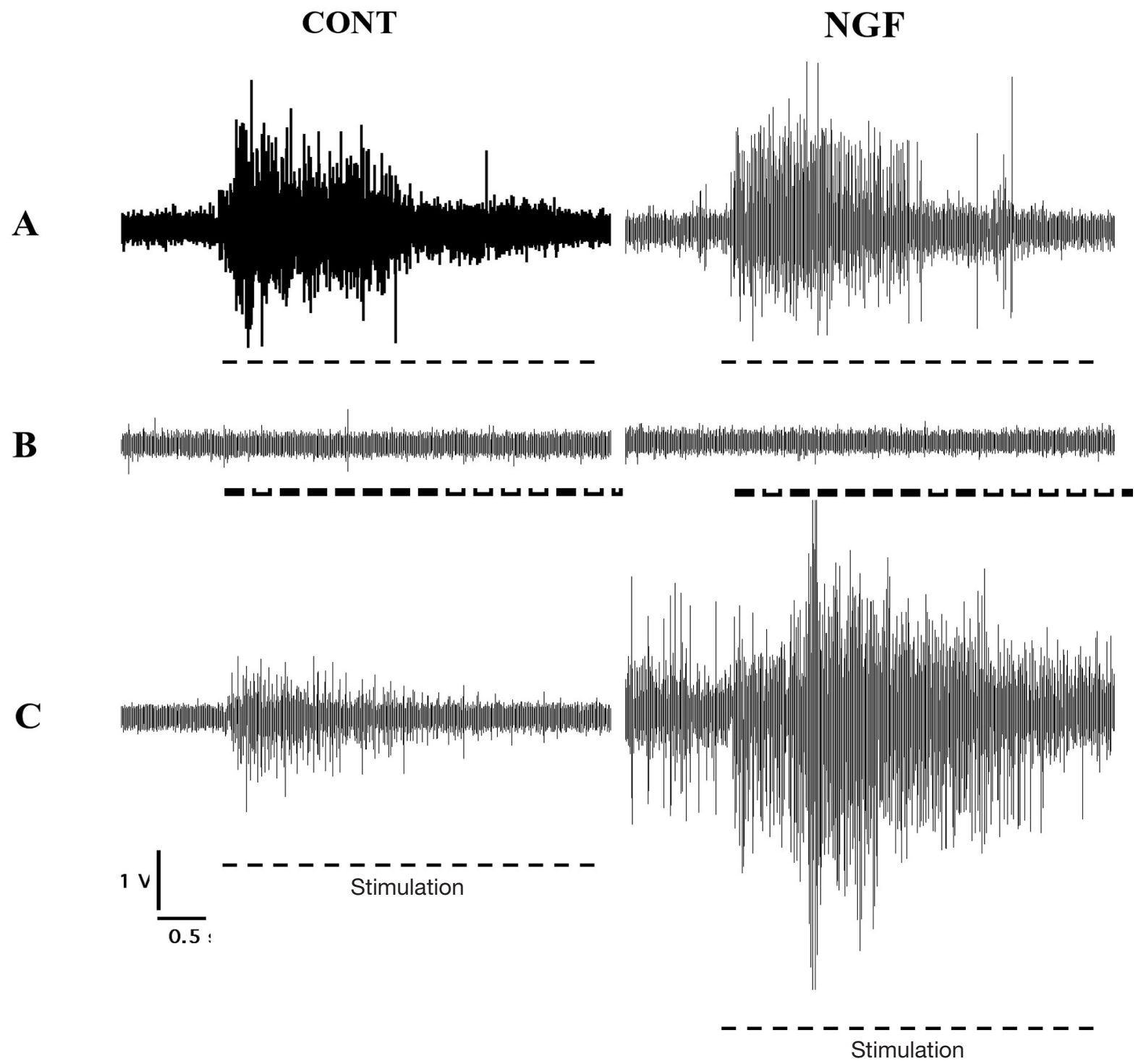

\section{PERSPECTIVES}

Un processus de régénération nerveuse précoce se produit même en l'absence de facteur neurotrophique exogène ${ }^{[5]}$, cependant l'application de la membrane fonctionnalisée par le NGF semble faciliter la régénération. L'étude sera poursuivie à deux et trois mois. De façon à mesurer la régénération, une évaluation quantitative des neurogrammes sera réalisée. De plus, un marquage fluorescent et une analyse morphologique des corps cellulaires des neurones sensitifs seront effectués. médecine buccale chirurgie buccale

VOL. $13, N^{\circ} 2$ 2007

page 105 


\section{RÉFÉRENCES}

1 - Hildebrand C, Fried K, Tuisku F, Johansson CS. Teeth and tooth nerves. Progr Neurobiol 1995; 45: 165-222.

2 - Robinson PP, Loescher AR, Yates JM, Smith KG. Current management of damage to the inferior alveolar and lingual nerves as a result of removal of third molars. $\mathrm{Br} \mathrm{J}$ Oral Maxillofac Surg 2004; 42: 285-92.

3 - Vodouhe C, Schmittbuhl M, Boulmedais F, Bagnard D, Vautier D, Schaaf P, Egles C, Voegel JC, Ogier J. Effect of functionalization of multilayered polyelectrolyte films on motoneuron growth. Biomaterials 2005; 26:545-54.
4 - Naftel JP, Richards LP, Pan M, Bernanke JM. Course and composition of the nerves that supply the mandibular teeth of the rat. Anat Rec 1999; 256:433-47.

5 - Chudler EH, Anderson LC, Byers MR. Trigeminal ganglion neuronal activity and glial fibrillary acidic protein immunoreactivity after inferior alveolar nerve crush in the adult rat. Pain 1997; 73:141-9. médecine buccale chirurgie buccale

VOL. $13, \mathrm{~N}^{\circ} 2$ 2007

page 106 